\title{
Parental Reflective Functioning as a Moderator for the Relationship Between Maternal Depression and Child Internalizing and Externalizing Problems
}

\author{
Saba Khoshroo ${ }^{1}$ D . Parisa Sadat Seyed Mousavi ${ }^{2}$ \\ Accepted: 18 June 2021 / Published online: 25 June 2021 \\ (c) The Author(s), under exclusive licence to Springer Science+Business Media, LLC, part of Springer Nature 2021
}

\begin{abstract}
Parental Reflective Functioning (PRF) refers to parents' capacity to view their child's and their own behavior considering inner mental states, like thoughts, desires, and intentions. This study's objective was to examine whether PRF acted as a moderator for the relationships between maternal depression and child internalizing and externalizing problems. The sample was comprised of 685 Iranian mothers with preschoolers ( 3 to 5 years old) and school-aged (6-10 years old) children. To assess PRF, the PRF questionnaire was used. Child internalizing and externalizing difficulties were assessed with the Child Behavior Checklist, and maternal depression was assessed with the Beck Depression Inventory- second version. Results indicated significant relationships between maternal depression and PRF (specifically pre-mentalizing and certainty in mental states). Also, mothers of school-aged children showed significantly higher levels of pre-mentalizing than mothers of preschoolers. Furthermore, certainty about mental states moderated the relationship between maternal depression and child externalizing behaviors in school-aged children. However, no moderating effect was found for pre-mentalizing or interest and curiosity in mental states in any age group.
\end{abstract}

Keywords Child externalizing problems $\cdot$ Child internalizing problems $\cdot$ Maternal depression $\cdot$ Mentalization $\cdot$ Parental reflective functioning

\section{Introduction}

In the last three decades, several studies have been conducted on maternal depression and its consequences. Depression is a relatively common disorder among women, and $25 \%$ of women experience depression clinically during their lifetime [1]. Moreover, $32 \%$ of mothers experience some symptoms of depression [2]. Mothers with depression tend to have more negative emotions and more irritability and hostility towards their children. They are more likely to discipline their children harshly and are more pessimistic about their parenting abilities [3]. Therefore, depression in mothers harms parent-child interactions, which may result in depression and

Saba Khoshroo

Sabakhoshroo@gmail.com

1 Family Research Institute, Shahid Beheshti University, Daneshjou blv, 1983969411 Tehran, Iran

2 Family Research Institute, Shahid Beheshti University, Tehran, Iran other psychological disorders, insecure attachment, emotion regulation problems, cognitive problems, and internalizing and externalizing problems in the child $[4,5]$. Goodman and colleagues [6] conducted a meta-analysis of 193 studies to better understand the effects of maternal depression on the child and to identify moderators for this relationship. The main conclusion was that mothers' depression, in all studies, was significantly correlated with internalizing and externalizing problems in their young children. Results indicated that the younger the child is, the stronger this relationship. Therefore, young children of depressed mothers are at higher risk for emotional and behavioral problems [6]. The following moderation effects were also found for this relationship: Family poverty as a moderator for both internalizing and externalizing problems, child gender as a moderator for only internalizing problems and father's absence as a moderator for only externalizing problems. It is also essential to consider a wide range of severity in depression, when examining parental behaviors because even mild depressive symptoms in mothers are associated with emotional and behavioral problems in their children $[7,8]$. 
Behavioral and emotional problems in children are usually divided into two broad areas: internalizing and externalizing problems, and each has different developmental consequences. While some problematic behaviors in preschoolers are developmentally appropriate, about $25 \%$ of young children show a clinically significant level of behavioral problems, and a subset of these children struggle with chronic behavioral problems [9]. Understanding the factors that predict the level of emotional and behavioral problems in childhood is essential because they are the goals of early intervention. Although previous studies have shown the relationship between mothers' depression and their children's behavioral problems, considering that not all children of mothers with depressive symptoms have behavioral problems of internalizing and externalizing [10], the study of moderating factors of this relationship is essential. It can pave the way for effective preventive and therapeutic interventions in this area.

One of the factors that can significantly affect the parent-child relationship is the mother's mentalization capacity. Mentalization or its operational term, Reflective Functioning (RF), is the ability to understand oneself and others' behaviors by considering mental states, including feelings, thoughts, desires, and goals [11]. Reflective functioning makes one 's or others' behaviors and emotional experiences more meaningful and predictable, especially in close and intimate relationships [12]. Moreover, Parental Reflective Functioning (PRF) refers to parents' reflective functioning toward themselves and their children, considering them as an independent unit, and the ability to meaningfully relate their child's behaviors to their inner states [13]. PRF is especially crucial in the early years of a child's life because infants and young children are entirely dependent on their caregivers to meet their needs due to cognitive and verbal capacity limitations. Those caregivers must be able to identify those needs, moods, and feelings correctly. Parents who have high PRF are curious about their child's mind and realize that moods can change over time and through different stages of development [11]. They are also sensitive and responsive to their child's needs, and high PRF promotes parental self-regulation, which leads to a better understanding of the parents' complicated relationship with the child. It also helps parents respond more confidently to their child's needs $[14,15]$. Subsequently, PRF plays a vital role in forming secure attachment, self-regulation, self-control, and emotion regulation in the child $[13,14,16]$. An intervention by Slade and colleagues [17] to increase PRF was performed on high-risk mothers during pregnancy until their child was two years old. This study showed that at one year of age, children whose mothers were in the intervention group had fewer externalizing and internalizing problems at age two. This study also showed that increasing PRF can mitigate drug use's adverse effects and early pregnancy on mothers
[17]. On the other hand, parents with lower PRF often fail to consider their infants as an independent unit with feelings and thoughts that influence their behaviors [18]. These parents have difficulty identifying their baby's mental states and may misinterpret them, such as "my baby is crying to bother me" [19]. Parents with low mentalization levels seem unaware of their own or their child's inner experiences and may also deny emotional experiences related to parenting. There is evidence that low PRF in mothers is associated with their child's increased vulnerability to mental disorders, and internalizing and externalizing problems in childhood, adolescence, and adulthood $[12,16,20]$. Several studies have shown that children of parents with high PRF exhibit less externalizing and internalizing problems [21-23].

Research has shown that psychological disorders, including depression, can affect the performance of RF [24]. Fischer-Kern and colleagues [25] conducted a study to assess the level of RF in women with major depression compared to non-depressed women. RF of women with depression was reported to be significantly lower than that of healthy women, and they were inferior at recognizing their own and others' internal states. The results also showed that the disease's duration, the number of referrals to medical centers, and the rate of cognitive distortion were significantly associated with RF levels, which means that the more chronic and severe the disease, the more RF is affected [25]. Also, Ramsauer and colleagues [26] concluded that depressed mothers were less likely than non-depressed mothers to focus on their child's needs and mental states. They also showed less insight and sensitivity towards the child's needs and wants and could not imagine their child as an independent entity separate from themselves and their emotions. Although this study did not directly measure PRF, the results obtained overlap with the dimensions of PRF. Depressed mothers also reported less flexibility, warmth, and acceptance towards their children [26]. On the other hand, some studies have been conducted on the relationship between depression and RF that are not in line with previous studies. For example, Cordes and colleagues [27] studied the mentalization capacity of mothers with postpartum depression symptoms, mothers with postpartum depression symptoms co-morbid with personality disorders and compared it with mothers without clinical symptoms. The results showed no significant difference between RF in any of the three groups. Commenting on the results, the researchers said that mild disorders such as postpartum depression do not necessarily change the mother's RF, and the severity of the disorder is important. In this sample, people with postpartum depression symptoms and not severe depressive disorder or chronic depression were studied [27]. Studies by Wong [28] and Vrieze [29] showed no difference between RF of depressed and non-depressed mothers, meaning that mothers with depression can respond to their child's needs correctly 
and promptly $[28,29]$. Previous research on the relationship between maternal depression and PRF do not seem to be consistent. Therefore, the study of the relationship between PRF and depression in mothers needs further research.

Some studies have suggested that without extensive intervention, PRF is a relatively stable construct throughout one's life $[30,31]$. Also, as mentioned, many studies found no relationship between maternal depression and PRF. Therefore, it can be possible for mothers with depressive symptoms to have high levels of RF. When a mother has high RF levels, she is better able to recognize and understand her own feelings and understand how those feelings impact her desire to respond to the child and how that, in turn, impacts the child. Many studies have linked high PRF to fewer behavior problems in children. As a result, it was hypothesized that for mothers with high levels of depressive symptoms, high levels of RF would act as a buffer to the negative effects of depressive symptoms on child development. Similarly, many studies have found PRF as a protective factor in the face of childhood adversities, including child sexual abuse [23], maternal depression [28], and difficult temperament [32], in relation to child outcomes. Therefore, this study aimed first to assess whether a significant relationship exists between maternal depression and PRF. Secondly, it aimed to investigate PRF's moderating effect on the relationship between maternal depression and child internalizing and externalizing problems. With regard to the research hypotheses, it was expected that (1) maternal depression would be positively associated with child internalizing and externalizing problems in both age groups, (2) PRF would be negatively associated with internalizing and externalizing problems in both age groups, (3) maternal depression would be negatively associated with PRF in both age groups and (4) PRF would moderate the relationship between maternal depression and child internalizing and externalizing problems in both age groups.

\section{Method}

\section{Participants}

In total, 685 Iranian mothers were included in this study that was conducted online. The mothers had children aged $3-10$. The majority (74.7\%) of the mothers were $30-40$ years old, followed by $20-30$ years old ( $16.3 \%$ ), over 40 years old (8.9\%), and under 20 years old $(0.1 \%)$. All mothers were Iranians residing in Iran. Participants varied in education levels and employment status, with the majority (82.8\%) having a bachelor's degree or higher, and fewer than 19\% earned a high school diploma or less. Also, the majority (65.8\%) of the participants were stay-at-home mothers, while $34.2 \%$ of them were employed. Just over half (51.7\%) of the children were female. The majority $(67.3 \%)$ of the children were between 3 to 6 years of age, $18 \%$ were $6-8$, and $14.7 \%$ were $8-10$ years old. Nearly half $(47.7 \%)$ of the children were an only child. Table 1 lists descriptive statistics of the sample and their children.

\section{Measures}

\section{Demographics}

Basic demographic information was collected at the time of the study. Participants provided information about their city of residency, age, job, education level, the father's education level, and their child's sex, order, and age.

Table 1 Descriptive statistics

\begin{tabular}{|c|c|}
\hline Maternal characteristics & $N(\%)$ \\
\hline \multicolumn{2}{|l|}{ Mother's age } \\
\hline Under 20 & $1(0.1)$ \\
\hline $20-30$ & $111(16.3)$ \\
\hline $30-40$ & $512(74.7)$ \\
\hline Over 40 & $61(8.9)$ \\
\hline \multicolumn{2}{|l|}{ Mother's education } \\
\hline Less than high school & $13(1.9)$ \\
\hline High school diploma & $105(15.3)$ \\
\hline Bachelor's degree & $354(51.7)$ \\
\hline Master's degree or higher & $213(31.3)$ \\
\hline \multicolumn{2}{|l|}{ Mother's job } \\
\hline Employed & $234(34.2)$ \\
\hline Stay at home & $451(65.8)$ \\
\hline \multicolumn{2}{|l|}{ Father's education } \\
\hline Less than high school; & $37(5.4)$ \\
\hline High school diploma & $150(21.9)$ \\
\hline Bachelor's degree & $254(37.1)$ \\
\hline Master's degree or higher & $244(35.6)$ \\
\hline Child characteristics & $N(\%)$ \\
\hline \multicolumn{2}{|l|}{ Age } \\
\hline $3-6$ & $461(67.3)$ \\
\hline $6-8$ & $123(18.0)$ \\
\hline $8-10$ & $101(14.7)$ \\
\hline \multicolumn{2}{|l|}{ Sex } \\
\hline Male & $331(48.3)$ \\
\hline Female & $354(51.7)$ \\
\hline \multicolumn{2}{|l|}{ Order of the child } \\
\hline Only child & $327(47.7)$ \\
\hline First child & $181(26.4)$ \\
\hline Second child & $147(21.5)$ \\
\hline Third child or above & $30(4.4)$ \\
\hline Total & $685(100)$ \\
\hline
\end{tabular}




\section{Parental Reflective Functioning}

PRF was measured using the Parental Reflective Functioning Questionnaire (PRFQ) [33]. The questionnaire consists of 18 items that measure PRF in three dimensions: pre-mentalizing modes, interest and curiosity in mental states, and certainty about mental states. It is important to note that PRFQ is a multidimensional construct, and each subscale taps on different features of PRF. Therefore, PRF is most accurate when assessed by each subscale separately, rather than the total score, as a single score cannot capture the complexity and multidimensionality of PRF [34]. Each question is scaled on a 7-point Likert, from 1 being strongly disagree, to 7 being strongly agree. The pre-mentalizing modes refer to the parent's non-mentalizing states and inability to effectively view the child's behavior as an expression of mental states, such as "My child cries around strangers to embarrass me." High scores on the pre-mentalizing subscale reflects the parents' tendency to make maladaptive attributions about the child, while low scores mean more accurate and effective attributions about the child's mental states. Interest and curiosity in mental states shows how much a parent is interested and exhibits curiosity in entering the child's inner world, with items such as "I try to see situations through the eyes of my child." Very high levels of interest and curiosity may indicate intrusive mentalizing, and very low levels of interest and curiosity may reveal a complete absent of interest in the child's inner world. Lastly, certainty about mental states refers to parent's ability to be certain about their child's mental state or, on the other hand, their inability to recognize the ambiguity of mental states, with questions such as "I always know what my child wants." An important aspect of certainty about mental states is that one should be aware of the opacity of mental states. In other words, very high score on the certainty of mental states subscale reflects no recognition of the opacity of mental states, which can be characterized as an impairment in mentalization. Low levels of certainty about mental states reflects the parents' complete confusion and uncertainty about the child's mental state. Luyten and colleagues [33, 34] demonstrated good internal consistencies for the PRFQ subscales, and the Persian translation of the PRFQ internal consistencies are as followed: Cronbach's $\alpha=0.67$ for pre-mentalizing modes, Cronbach's $\alpha=0.74$ for certainty about mental states, and Cronbach's $\alpha=0.72$ for interest and curiosity in mental states [35]. The three PRFQ subscales' internal consistencies in the current study were as follows: Cronbach's $\alpha=0.60$ for pre-mentalizing modes, Cronbach's $\alpha=0.77$ for certainty about mental states, and Cronbach's $\alpha=0.68$ for interest and curiosity in mental states.

\section{Beck Depression Inventory- Second Version}

The BDI-II was used to assess self-report maternal depression levels [33]. BDI-II consists of 21 questions that measure different aspects of depressive symptoms, with each question having four possible options ranging in the severity of the symptoms. Each question receives a score between 0 and 3, with three being most severe, and the responses are summed for a final score. The BDI-II has shown high internal consistency Cronbach's $\alpha=0.91$ [36]. The internal consistency of the Persian translation of the BDI-II is Cronbach's $\alpha=0.87$ [37] and in the current study was Cronbach's $\alpha=0.92$.

\section{The Child Behavior Checklist (CBCL/1.5-5 and $\mathrm{CBCL} / 6-18$ )}

Both parent-report versions of CBCL [38, 39] were used to assess child behavior problems and social competencies. CBCL/1.5-5 consists of 99 questions while CBCL/6-18 has 113 questions, and both versions are scored on a three-point Likert scale, with $0=$ never occurs, $1=$ occurs sometimes, and $2=$ occurs often. Items are summed to yield scores for different behavior problems and global scores. In the current study, the two global scales of internalizing and externalizing problems were used. Both versions of the Persian translation of the CBLC have shown good to high internal consistencies [40], and the internal consistencies of the CBCL for both versions in the current study were Cronbach's $\alpha=0.94$.

\section{Procedure}

Online versions of the Persian translations of the questionnaires were developed through Google Forms, and due to quarantine restrictions, were shared solely via social media (Instagram, Twitter, and Telegram). The link to the questionnaire was shared on different Instagram accounts which mostly targeted mothers as their main audience. Two accounts were child psychology pages, and one account was a kids photography studio. Moreover, the link to the questionnaire was also shared via an anonymous account on Twitter and Telegram, asking for the participation of mothers with a 3- to 10-year-old child. 690 mothers with children aged 3-10 completed the one-time questionnaires, but five were concluded to be outliers in the multivariate test, which were excluded from further analyses. The participants gave informed consent at the beginning of the questionnaire and were able to withdraw at any time. The board of Family Research Institute at Shahid Beheshti University, Tehran, Iran, approved the procedure. 


\section{Results}

\section{Preliminary Analyses}

All study variables in both age groups were tested for univariate and multivariate outliers. Among 690 initial responses, five were concluded to be outliers in the multivariate test, which were excluded from further analyses. Skewness and kurtosis were analyzed to assess normality, and both age groups were concluded to have a normal distribution. Furthermore, to check for collinearity, variance inflation factor and tolerance were assessed for maternal depression and the three sub-scales of PRFQ in predicting internalizing and externalizing problems in both age groups. No collinearity was found.

\section{Correlational Analyses}

To assess relationships among study variables, correlational analyses were used. Regarding the first hypothesis: Maternal depression was significantly and positively correlated with externalizing $(r=0.425, P<0.001)$ and internalizing $(r=0.417, P<0.001)$ problems in preschoolers, as well as externalizing $(r=0.393, P<0.001)$ and internalizing $(r=0.464, P<0.001)$ problems in school-aged children. Regarding the second hypothesis: Maternal pre-mentalizing modes in mothers with a preschooler, and internalizing problems and externalizing problems in the child were significantly and positively correlated, $r=0.404, P<0.001$, $r=0.431, P<0.001$, respectively. Similarly, maternal prementalizing modes in mothers with a school-aged child and internalizing problems and externalizing problems in the child were also significantly and positively correlated, $r=0.302, p<0.001, r=0.489, p<0.001$, respectively. Certainty in mental states was significantly and negatively associated with internalizing $(r=-0.124, p<0.01)$ and externalizing $(r=-0.158, p<0.01)$ problems in preschoolers, but not school-aged children. Interest and curiosity in mental states was significantly and positively correlated only with internalizing problems $(r=0.183, p<0.01)$ in school-aged children. Regarding the third hypothesis: Correlation analyses showed that maternal depression was significantly and positively associated with pre-mentalizing $r=0.379$, $p<0.001$ and was significantly and negatively associated with certainty in mental states $r=-0.152, p<0.001$. No significant relationship was found between maternal depression and interest and curiosity in mental states. The correlation analyses among study variables are shown in Table 2.

\section{MANOVA on PRFQ Subscales and Descriptive Measures}

In order to assess differences in PRF levels in regard to characteristic differences among the sample, MANOVA was performed on the PRFQ subscales with all descriptive measures. Means and standard deviations of PRFQ for child's age and mother's education are reported in Tables 3 and 4, respectively. A significant effect of the child's age was found on pre-mentalizing $\left(F_{(1,685)}=12.123, P<0.001\right)$, with mothers of preschoolers scoring significantly lower than mothers of school-aged children. Pre-mentalizing was also affected by mothers' education $\left(F_{(1,685)}=23.761, P<0.001\right)$, with mothers holding a high school diploma or less scoring

Table 3 Analysis of variance for child's age group

\begin{tabular}{lllllll}
\hline Child's age group & $3-5$ & & & $6-10$ & \multirow{2}{*}{$F(1,684)$} \\
\cline { 2 - 3 } & $M$ & $S D$ & & $M$ & $S D$ & \\
\hline PRFQ_PM & 12.16 & 5.10 & & 13.67 & 5.64 & $12.123^{* * * *}$ \\
PRFQ_CM & 27.58 & 6.58 & & 27.90 & 5.39 & .419 \\
PRFQ_IC & 35.58 & 5.38 & & 34.95 & 5.21 & 2.074 \\
\hline
\end{tabular}

$P R F Q \_P M$ Pre-Mentalizing, $P R F Q \_C M$ Certainty about mental states, $P R F Q \_I C$ Interest and Curiosity

*** $p<.001$

Table 2 Ratings and correlations among PRF (PRFQ sub-scales), Depression (BDI), and child internalizing and externalizing problems (CBCL)

\begin{tabular}{|c|c|c|c|c|c|c|c|c|c|c|c|}
\hline Variable & $n$ & $M$ & $S D$ & 1 & 2 & 3 & 4 & 5 & 6 & 7 & 8 \\
\hline 1. Pre-mentalizing & 685 & 12.6 & 5.39 & - & & & & & & & \\
\hline 2. Certainty & 685 & 27.7 & 6.52 & -.049 & - & & & & & & \\
\hline 3. Interest and Curiosity & 685 & 35.4 & 5.32 & $-.078^{*}$ & $.252^{* *}$ & - & & & & & \\
\hline 4. Internalizing problems $3-5$ & 461 & 9.5 & 7.20 & $.404^{* *}$ & $-.124^{* *}$ & -.061 & - & & & & \\
\hline 5. Externalizing problems $3-5$ & 461 & 11.2 & 7.24 & $.431^{* *}$ & $-.158^{* *}$ & -.071 & $.662^{* *}$ & - & & & \\
\hline 6. Internalizing problems $6-10$ & 223 & 11.2 & 6.60 & $.302^{* *}$ & .023 & $.183^{* *}$ & $\mathrm{c}$ & $\mathrm{c}$ & - & & \\
\hline 7. Externalizing problems 6-10 & 224 & 11.9 & 7.84 & $.489^{* *}$ & -.065 & .089 & $\mathrm{c}$ & $\mathrm{c}$ & $0.542^{* *}$ & - & \\
\hline 8. Depression & 685 & 11.5 & 10.22 & $0.379^{* *}$ & $-.152^{* *}$ & -.028 & $.417^{* * *}$ & $.425^{* *}$ & $.464^{* *}$ & $.393^{* *}$ & - \\
\hline
\end{tabular}

$c$ Cannot be computed because at least one of the variables is constant

${ }^{*} p<.05 .{ }^{* *} p<.01$ 
Table 4 Analysis of variance for mother's education

\begin{tabular}{lllllll}
\hline $\begin{array}{l}\text { Mother's edu- } \\
\text { cation level }\end{array}$ & $\begin{array}{l}\text { High school } \\
\text { diploma or less }\end{array}$ & & \multicolumn{2}{l}{$\begin{array}{l}\text { Bachelor's degree } \\
\text { or higher }\end{array}$} & $F(1,685)$ \\
\cline { 2 - 3 } \cline { 5 - 6 } & $M$ & $S D$ & & $M$ & $S D$ & \\
\hline PRFQ_PM & 14.81 & 6.27 & & 12.20 & 5.07 & $23.761^{* * *}$ \\
PRFQ_CM & 27.84 & 7.40 & & 27.63 & 6.33 & .105 \\
PRFQ_IC & 35.46 & 5.14 & & 35.35 & 5.32 & .038 \\
\hline
\end{tabular}

$P R F Q \_P M$ Pre-Mentalizing, $P R F Q \_C M$ Certainty about mental states, $P R F Q \_I C$ Interest and Curiosity

${ }^{* * *} p<.001$

significantly higher than mothers with a bachelor's degree or higher. No difference in PRF indices were found in regard to mother's age, mother's job, father's job, child's sex, or child's order.

\section{Results of Moderation Analyses}

Hierarchical regression analyses were conducted using SPSS, and graphs of moderations were conducted using PROCESS [41]. A total of 12 regression analyses were conducted. In the first block, maternal depression and the 3 PRFQ subscales were entered as independent variables, while externalizing and internalizing problems were entered as dependent variables. In the second block, interactions of maternal depression and the 3 PRFQ subscales were added as the independent variables. Separate analyses were conducted for each age group, each subscale, and each behavior problem (internalizing and externalizing), totaling in 12 analyses. Results revealed that certainty in mental states moderated the relationship between maternal depression and child externalizing problems in school-aged children. In the first block, maternal depression $(T=6.274, \beta=0.391, P=0.001)$ and certainty in mental states $(T=-0.324, \beta=-0.02, P=0.746)$ were entered as predictor variables. Maternal depression significantly predicted externalizing problems, and, in the second block, their interaction $(\beta=0.595,95 \%$ CI [0.001, 0.032], $T=2.123, p<0.05)$ significantly predicted externalizing problems. As shown in Fig. 1, when certainty in mental states is low, there is a weak correlation between maternal depression and child externalizing problems. As certainty in mental states increases, the relationship between maternal depression and child externalizing problems becomes stronger. None of the other subscales of PRFQ showed any significant moderation effect in any age group.

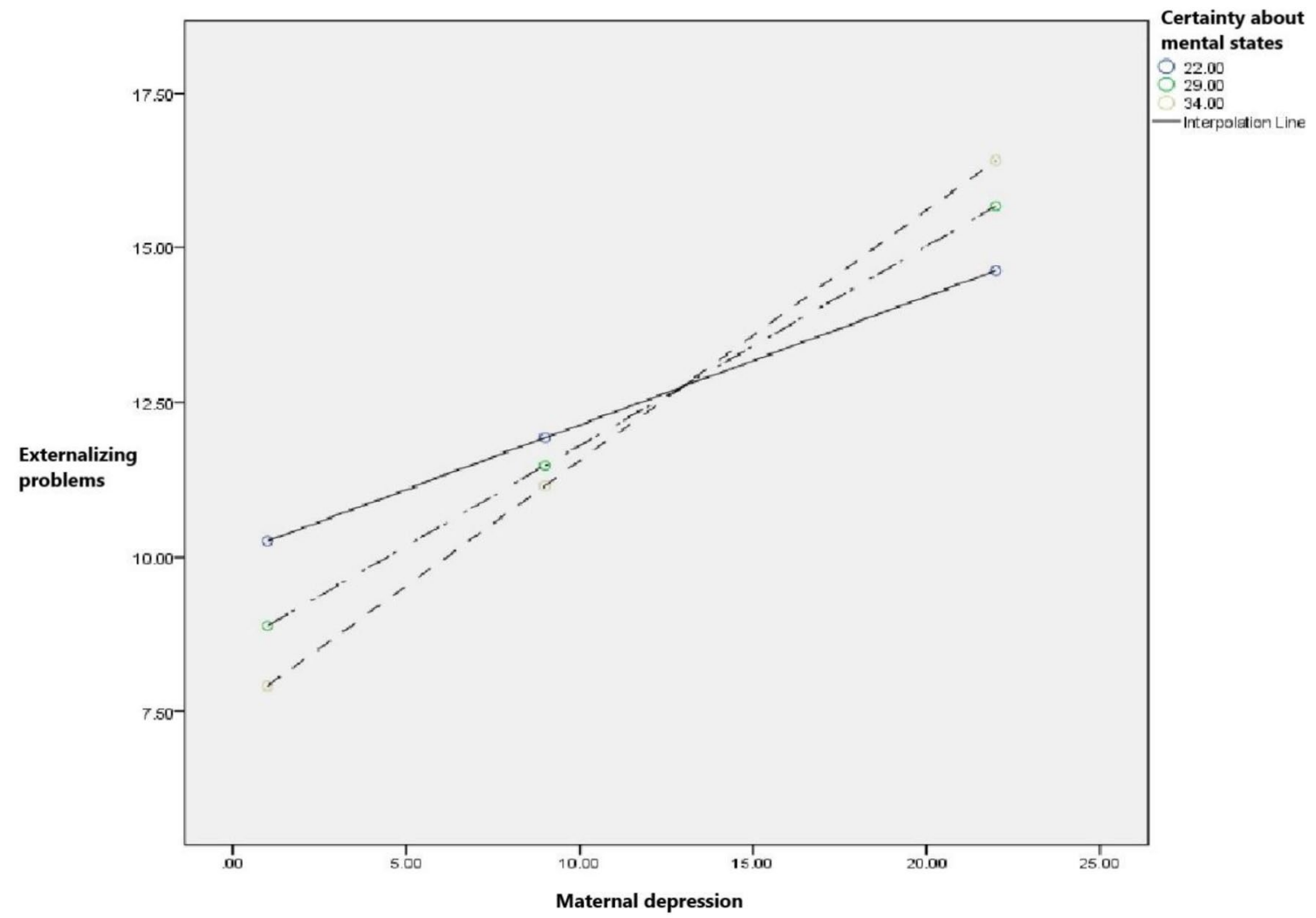

Fig. 1 Interaction between certainty in mental states and maternal depression 


\section{Discussion}

The current study's purpose was to conduct an initial exploration of whether PRF and maternal depression are associated, and whether PRF moderates the relationship between maternal depression and child internalizing and externalizing problems. Results were in line with the first hypothesis. As expected, depression was significantly and positively correlated with internalizing and externalizing problems in children in both age groups [6, 42]. As for the second hypothesis, similar to prior research pre-mentalizing modes were significantly and positively correlated with externalizing and internalizing problems in both age groups. Therefore, children of mothers with high levels of pre-mentalizing, which can be said is the lack of effective $\mathrm{RF}$, exhibit more internalizing and externalizing problems than children with mothers with low levels of pre-mentalizing. Additionally, certainty about mental states was also significantly and negatively correlated with externalizing and internalizing problems in preschoolers, indicating that whenever parents are less certain and more confused about their child's mental state, the child is more likely to show internalizing and externalizing problems. Lastly, interest and curiosity in mental states was significantly and positively correlated with internalizing problems in school-aged children.

Externalizing problems are an immature attempt for young children to show distress, and mothers with higher RF levels tend to look more into the reason for the behavior rather than the behavior itself. Therefore, they are more likely to engage in a constructive conversation with the child about his/her needs and intentions. This conversation would help the child regulate his/her emotions when faced with overwhelming and emotionally charged situations and not use aggressive or rule-breaking behavior. On the other hand, mothers with lower mentalization levels tend to believe that the child's behavior is intentional and in his/her control. Therefore, the mother becomes distressed and may punish the child, which in turn may increase the undesired externalizing behavior [23, 43]. The significant relationship between pre-mentalizing modes and internalizing problems in children aged 3 to 10 years indicates that parents' ability to effectively mentalize and make adaptive attributions, provides a sense of security and trust in the child. If a mother with low PRF does not recognize or misinterprets her child's inner feelings and moods, she may ignore her child's needs, which may result in feelings of neglect, helplessness, mistrust, and anxiety, and consequently may lead to internalizing problems in the child [20, 44]. In other words, continuous mentalization and reflection upon underlying causes of behavior is crucial for developing emotion regulation, self-regulation, and a sense of security in children $[45,46]$, which in turn help decrease internalizing and externalizing problems [47, 48]. Previous research has shown similar results, indicating significant relationships between indices of PRF and internalizing and externalizing problems in preschoolers and school-aged children [21, 44]. Moreover, studies assessing PRF have found a robust association with the development of secure attachment in the child [49-51], and secure attachment has been strongly linked to less internalizing and externalizing problems in children [52-55].

Parents who are overly ambiguous about their child's mental states and needs may not be able to correctly identify their child's needs or they may even deny the existence of an inner world altogether, such as believing that "my child is too young to think." This kind of confusion and ambiguity of the mother in identifying her child's inner motivations leads to the mother's withdrawal behaviors, which is one of the risk factors of anxiety in children [56]. One possible explanation for the positive correlation between interest and curiosity in mental states and internalizing problems in school-aged children could be that high levels of interest can lead to intrusive mentalizing [34], meaning too much interruption and involvement in the child's inner world and daily life. School-aged children develop a growing desire for autonomy, and intrusive involvement and overprotection from their parents may result in anxious and withdrawal behaviors [57, 58].

The third hypothesis of this study was to assess whether maternal depression is associated with PRF. Pre-mentalizing modes was found to be significantly higher, while certainty in mental states was significantly lower in depressed mothers than non-depressed mothers. This suggests that depressed mood in mothers is associated with difficulties in affectively reading a child's mind and being somewhat certain about it. A study by Krink and colleagues [59], which similarly used the PRFQ, found similar results. They found that women with postpartum depression scored higher on prementalizing modes, and the higher the degree of depression, the higher the mothers' inability to mentalize. Similar to the present study, no significant relationship was found between mothers' depression and their scores on interest and curiosity in mental states [59]. It can be concluded that a depressed mood does not necessarily change the extent to which mothers are interested and curious to know about their child's inner states, but rather impairs mothers' capacity to reflect on themselves and others, which makes them more likely to have a distorted perception of themselves and their child. To better understand this phenomenon, Luyten and Blatt [60] found that in depression, pre-mentalizing modes occur that hinder the individual's ability to mentalize effectively. For example, according to the theory of development of psychic reality, in a state of psychic equivalence, inner experiences are considered equal to the outside world, where 
psychological pain is experienced as bodily pain, and criticism of others feels like a physical attack. In the pretend mode, thoughts and emotions are far from reality, usually expressed exaggeratedly. This includes self-blame, repeated blame of others, and high emotional irritability, common among people with depression. This is seen in the PRFQ: "My child cries around strangers to embarrass me," which is one of the questions that measure pre-mentalizing modes. In the teleological mode, only visible behaviors are considered real, measured in the PRFQ as "the only time I am sure my child loves me is when he smiles at me." As a result, it can be concluded that mothers with depression are more likely to stay in one or more of the pre-mentalizing modes and score higher on the sub-scales of pre-mentalizing in the PRFQ [60]. Also, cognitive symptoms, including inability to concentrate, make decisions, and pay attention, are the major complaints of people with depression [61]. Therefore, mothers with depressed moods are less able to focus on their child's signs of upset and make a definite decision about the underlying causes; instead, they are in an aura of ambiguity and confusion. Moreover, mothers with depressed moods are less likely to tune into their child's emotions, communicate verbally, and be sensitive and responsive [3]. All of the above can be factors that reduce mothers' ability to guess the inner states and understand the needs and motivations of their young child correctly and effectively, as a result of which they can be less certain of their child's inner states.

The objective of this study was to investigate whether PRF acted as a moderator for the relationship between maternal depression and child internalizing and externalizing problems. Certainty about mental states was found to moderate the relationship between maternal depression and externalizing problems in 6 to 10-year-old children. In other words, when certainty in mental states is moderate or high, the relationship between maternal depression and externalizing problems is more pronounced, which indicates the moderating effect of this variable. No moderating effect was found for pre-mentalizing modes or interest and curiosity in mental states in any age group. To explain this result, as mentioned previously, when a mother is in the pre-mentalizing modes, she cannot effectively realize the emotions, intentions, and thoughts behind her child's behavior. This is often accompanied by misinterpretations and sometimes cruel interpretations of her child's intentions. In other words, because of the cognitive distortions and pessimism that follow depression, depressed mothers often have a negative and hopeless view of themselves and their child. For example, a depressed mother may attribute her child's aggressive behaviors to unrealistic reasons, such as that he/she is seeking revenge or that "my child is inherently evil" [33]. For this reason, it can be said that when a mother with depression has high certainty about these irrational thoughts, she becomes infuriated herself and may ignore the psychological needs of her child that caused the aggressive behavior and turn more to harsh disciplinary methods that aggravate the behavior. Therefore, it seems that depressed mothers with lower levels of certainty in mental states insist less on their misinterpretations of their child's behavior, and consequently, act less upon them. As a result, the relationship between maternal depression and externalizing problems is weakened.

One of the reasons that other moderating relationships were not significant could that the study used a community sample whose depressive symptoms ranged from mild to moderate and rarely severe. Among the sample, only 36 children aged 3 to 5 years and 19 children aged 6 to 10 years showed behavioral and emotional problems clinically (with a $\mathrm{T}$ score above 65). Due to the low sample size and error in moderation analysis, it was not possible to perform the analysis again with only mothers with moderate to high depression scores. Community sample research have shown to have great benefits, like high generalizability, enhanced relevance and usefulness, the ability to build a broad body of knowledge related to well-being and the ability to bridge the cultural gaps that may exist [62]. However, studies have shown that the relationship between RF, depression, and mediating variables depend on the severity of depression and studying these constructs in a clinical setting may yield different results $[59,63]$. It is important to note that PRF is a relatively new field in mentalization research and needs further validation, especially the PRFQ and its validations in eastern countries. Replications are crucial to better understand PRF's mechanisms and how it works explicitly in the parent-child dyad.

Additional findings suggest that mothers with children aged 6 to 10 years stay in pre-mentalizing stages significantly more than mothers with children aged 3 to 5 years. This indicates that mothers with school-aged children have a more difficult time reading their child's mind and feelings than mothers with preschoolers. Consistent with prior work [64], it can be argued that when children get older, they can more easily control the amount and type of emotions they express, while younger children have more transparent and predictable needs. As a result, it is possible for an older child to self-regulate in a way that misleads parents. In line with their developmental needs, school-aged children gain more experience outside of the family environment, with teachers and peers. The child's interactions with teachers and peers can affect his thoughts and feelings about him/herself and the world and, consequently, influence his or her behavior [65]. A parent who is unaware of these experiences and interactions, and the child is reluctant to share them for some reason, may be less able to interpret their child's behaviors and may have distorted perception. Furthermore, the results showed that mothers with high school education or lower scored significantly higher on pre-mentalizing modes than mothers with a bachelor's degree or higher education 
levels. This result is consistent with other studies that also used the PRFQ, indicating that maternal education (at least three years after graduation) has an inverse and significant relationship with pre-mentalizing modes [33]. This finding is consistent with theories that argue that economically and socially inferior individuals are also deprived of the ability to mentalize, as a result of which most of the world is considered pessimistic and hostile [66].

\section{Limitations and Future Directions}

The present study contributes to understanding the interplay among maternal depression, PRF, and child behavioral problems and emphasizes the critical relationship between maternal depression and PRF. Nevertheless, some limitations and future directions must be acknowledged. This study's data collection was completed during the second month of quarantine in Iran due to the Covid-19 pandemic. The nonfamiliar challenges of quarantine may have influenced maternal depressive moods and children's emotional and behavioral problems. It is important to be cautious when interpreting the results. A limitation in internal validity can be seen for the relatively low reliabilities of sub-scales of pre-mentalizing $(\alpha=0.60)$ and interest and curiosity in mental states $(\alpha=0.68)$ in the Persian version. In another study using the Persian translation of PRFQ, both subscales showed relatively lower reliabilities than certainty in mental states: $\alpha=0.42$ for pre-mentalizing and $\alpha=0.64$ for interest and curiosity in mental states [67]. A similar issue has been seen in the German translation of PRFQ [59]. This can be attributed to different cultures and how PRFQ translation might not necessarily be a good cultural fit. Additionally, the low reliability could be explained by the sample's homogeneity, which resulted in lower variances. The present study is among the first studies to use the Persian translation of the PRFQ. Many more studies are needed to assess the validity and reliability of this questionnaire in the Iranian population. Replicating the current study with clinically depressed mothers and assessing PRF via interviews may yield different results.

\section{Summary}

The aim of this study was to examine whether PRF acted as a moderator for the relationships between maternal depression and child internalizing and externalizing problems. The sample was comprised of 685 Iranian mothers with preschoolers ( 3 to 5 years old) and school-aged (6-10 years old) children. As hypothesized, maternal depression was significantly associated with child internalizing and externalizing problems in both age groups. Also, PRF was associated with internalizing and externalizing problems in both age groups. Results indicate significant relationships between maternal depression and PRF (specifically pre-mentalizing and certainty in mental states). Also, mothers of school-aged children showed significantly higher levels of pre-mentalizing than mothers of preschoolers. Furthermore, certainty about mental states moderated the relationship between maternal depression and child externalizing behaviors in schoolaged children. However, no moderating effect was found in pre-mentalizing or interest and curiosity in mental states in any age group. These findings provide valuable information about the importance of depression and mentalization research in the Iranian population.

Author Contributions SK: Conceptualization, methodology, investigation (data collection), formal analysis, writing- original draft, writingreview, and editing. PSSM: Supervision, validation, writing-review, and editing. All authors agreed to be accountable for all aspects of the work in ensuring that questions related to the accuracy or integrity of any part of the work are appropriately investigated and resolved.

Funding This research did not receive any specific grant from funding agencies in the public, commercial, or not-for-profit sectors.

\section{Declarations}

Conflict of Interest The authors declare that there is no conflict of interest.

Ethical Approval This paper is adapted from a master's thesis by Saba Khoshroo titled "Parental Reflective Functioning as a Moderator for Relationship between Maternal Depression and Child Internalizing and Externalizing problems." The thesis, no. 90-10-904, has been written under the supervision of Dr. Parisa Sadat Seyed Mousavi and approved by the committee at the Family Research Institute of Shahid Beheshti University.

Reasearch Involving Human and Animal Rights All procedures performed in studies involving human participants were in accordance with the ethical standards of the institutional and/or national research committee and with the 1964 Helsinki declaration and its later amendments or comparable ethical standards.

Informed Consent Informed consent was obtained from all individual participants included in the study.

\section{References}

1. Goodman SH, Gotlib IH (2002) Children of depressed parents: mechanisms of risk and implications for treatment. Washington, DC.

2. Wang L, Wu T, Anderson JL, Florence JE (2011) Prevalence and risk factors of maternal depression during the first three years of child rearing. J Womens Health 20(5):711-718

3. Lovejoy MC, Graczyk PA, O'Hare E, Neuman G (2000) Maternal depression and parenting behavior: a meta-analytic review. Clin Psychol Rev 20(5):561-592 
4. Cicchetti D, Rogosch FA, Toth SL (1998) Maternal depressive disorder and contextual risk: contributions to the development of attachment insecurity and behavior problems in toddlerhood. Dev Psychopathol 10(2):283-300

5. Shaw DS, Hyde LW, Brennan LM (2012) Early predictors of boys' antisocial trajectories. Dev Psychopathol 24(3):871-888

6. Goodman SH, Rouse MH, Connell AM, Broth MR, Hall CM, Heyward D (2011) Maternal depression and child psychopathology: a meta-analytic review. Clin Child Fam Psychol Rev 14(1):1-27

7. Choe DE, Olson SL, Sameroff AJ (2013) Effects of early maternal distress and parenting on the development of children's self-regulation and externalizing behavior. Dev Psychopathol 25(2):437-453

8. West AE, Newman DL (2003) Worried and blue: mild parental anxiety and depression in relation to the development of young children's temperament and behavior problems. Parent Sci Pract 3(2):133-154

9. Campbell SB (1995) Behavior problems in preschool children: a review of recent research. J Child Psychol Psychiatry 36(1):113-149

10. Weissman MM, Warner V, Wickramaratne P, Moreau D, Olfson M (1997) Offspring of depressed parents: 10 years later. Arch Gen Psychiatry 54(10):932-940

11. Fonagy P, Steele M, Steele H, Moran GS, Higgitt AC (1991) The capacity for understanding mental states: the reflective self in parent and child and its significance for security of attachment. Infant Ment Health J 12(3):201-218

12. Bateman A, Peter (Psychoanalysis Unit Fonagy, \& Fonagy, $\mathrm{P}$ (2004) Psychotherapy for borderline personality disorder. Oxford University Press, Oxford, pp 529-532

13. Slade A (2005) Parental reflective functioning: an introduction. Attach Hum Dev 7(3):269-281

14. Sharp C, Fonagy P (2008) The parent's capacity to treat the child as a psychological agent: constructs, measures and implications for developmental psychopathology. Soc Dev 17(3):737-754

15. Suchman NE, DeCoste C, Leigh D, Borelli J (2010) Reflective functioning in mothers with drug use disorders: implications for dyadic interactions with infants and toddlers. Attach Hum Dev 12(6):567-585

16. Bouchard MA, Target M, Lecours S, Fonagy P, Tremblay LM, Schachter A, Stein H (2008) Mentalization in adult attachment narratives: reflective functioning, mental states, and affect elaboration compared. Psychoanal Psychol 25(1):47

17. Slade A, Holland ML, Ordway MR, Carlson EA, Jeon S, Close N, Mayes LC, Sadler LS (2019) Minding the baby®: enhancing parental reflective functioning and infant attachment in an attachment-based, interdisciplinary home visiting program. Dev Psychopathol, 1-15.

18. Rutherford HJ, Booth CR, Luyten P, Bridgett DJ, Mayes LC (2015) Investigating the association between parental reflective functioning and distress tolerance in motherhood. Infant Behav Dev 40:54-63

19. Burkhart ML, Borelli JL, Rasmussen HF, Brody R, Sbarra DA (2017) Parental mentalizing as an indirect link between attachment anxiety and parenting satisfaction. J Fam Psychol 31(2):203

20. Ensink K, Normandin L, Plamondon A, Berthelot N, Fonagy P (2016) Intergenerational pathways from reflective functioning to infant attachment through parenting. Can J Behav Sci 48(1):9

21. Kapeleris A (2014) Children's socio-emotional development and adjustment: role of maternal trauma, mentalization and parenting style.

22. Ordway MR, Sadler LS, Dixon J, Close N, Mayes L, Slade A (2014) Lasting effects of an interdisciplinary home visiting program on child behavior: preliminary follow-up results of a randomized trial. J Pediatr Nurs 29(1):3-13
23. Ensink K, Bégin M, Normandin L, Fonagy P (2017) Parental reflective functioning as a moderator of child internalizing difficulties in the context of child sexual abuse. Psychiatry Res 257:361-366

24. Katznelson H (2014) Reflective functioning: a review. Clin Psychol Rev 34(2):107-117

25. Fischer-Kern M, Fonagy P, Kapusta ND, Luyten P, Boss S, Naderer A, Blüml V, Leithner K (2013) Mentalizing in female inpatients with major depressive disorder. J Nerv Ment Dis 201(3):202-207

26. Ramsauer B, Lotzin A, Mühlhan C, Romer G, Nolte T, Fonagy P, Powell B (2014) A randomized controlled trial comparing Circle of Security Intervention and treatment as usual as interventions to increase attachment security in infants of mentally ill mothers: study protocol. BMC Psychiatry 14(1):24

27. Cordes K, Smith-Nielsen J, Tharner A, Katznelson H, Steele H, Væver M (2017) Reflective functioning in postpartum depressed women with and without comorbid personality disorder. Psychoanal Psychol 34(4):414

28. Wong KM (2012) A study of the effect of maternal depressive symptoms on the mother-infant relationship and protective effect of maternal reflective functioning (Master's Thesis)

29. Vrieze DM (2011) The role of parental reflective functioning in promoting attachment for children of depressed mothers in a toddler-parent psychotherapeutic intervention (doctoral dissertation). Retrieved from Digital Conservancy, University of Minnesota

30. Steele H, Steele M (2008) On the origins of reflective functioning. In: Busch F (ed) Mentalization: theoretical considerations, research findings, and clinical implications. Routledge Publishing, London, pp 133-158

31. Wong KML (2016) A study of stability: maternal reflective functioning from pregnancy to seven months postpartum.

32. Wong K, Stacks AM, Rosenblum KL, Muzik M (2017) Parental reflective functioning moderates the relationship between difficult temperament in infancy and behavior problems in toddlerhood. Merrill-Palmer Q 63(1):54-76

33. Luyten P, Mayes LC, Nijssens L, Fonagy P (2017) The parental reflective functioning questionnaire: development and preliminary validation. PloS One 12(5):6218

34. Luyten P, Nijssens L, Fonagy P, Mayes LC (2017) Parental reflective functioning: theory, research, and clinical applications. Psychoanal Study Child 70(1):174-199

35. Goudarzi Z (2020) The mediation role of emotion regulation between the relationship of maternal reflective functioning and children's internalizing and externalizing disorders. (Unpublished master's thesis). Shahid Beheshti University, Tehran, Iran.

36. Beck, A. T., Steer, R. A., \& Brown, G. (1996). Beck depression inventory-II. Psychological Assessment.

37. Ghassemzadeh H, Mojtabai R, Karamghadiri N, Ebrahimkhani N (2005) Psychometric properties of a Persian-language version of the Beck Depression Inventory-Second edition: BDI-II-PERSIAN. Depress Anxiety 21(4):185-192

38. Achenbach TM, Rescorla LA (2001) Manual for the ASEBA school-age forms and profiles. University of Vermont. Research Center for Children, Youth \& Families, Burlington, VT

39. Achenbach TM, Rescorla LA (2000) Manual for the ASEBA preschool forms and profiles. University of Vermont, Research Center for Children, Youth, \& Families, Berlington, VT

40. Tehrani-Doost M, Shahrivar Z, Pakbaz B, Rezaie A, Ahmadi F (2011) Normative data and psychometric properties of the child behavior checklist and teacher rating form in an Iranian community sample. Iran J Pediatr 21(3):331

41. Hayes AF (2012) PROCESS: a versatile computational tool for observed variable mediation, moderation, and conditional process modeling. 
42. Wagner KM, Valdez CR (2020) The relationship between maternal depression, externalizing and internalizing problems in children, and caregiving burden in urban low-income ethnic and racial minority families. Child Psychiatry Hum Dev 51(3):390-398

43. Gershoff ET (2002) Corporal punishment by parents and associated child behaviors and experiences: a meta-analytic and theoretical review. Psychol Bull 128(4):539

44. Esbjørn BH, Pedersen SH, Daniel SI, Hald HH, Holm JM, Steele $H$ (2013) Anxiety levels in clinically referred children and their parents: examining the unique influence of self-reported attachment styles and interview-based reflective functioning in mothers and fathers. Br J Clin Psychol 52(4):394-407

45. Fonagy P, Target M (1997) Attachment and reflective function: their role in self-organization. Dev Psychopathol 9(4):679-770

46. Borelli JL, Lai J, Smiley PA, Kerr ML, Buttitta K, Hecht HK, Rasmussen HF (2020) Higher maternal reflective functioning is associated with toddlers' adaptive emotion regulation. Infant Ment Health J. https://doi.org/10.1002/imhj.21904

47. Brenning KM, Soenens B, Braet C, Bosmans GUY (2012) Attachment and depressive symptoms in middle childhood and early adolescence: testing the validity of the emotion regulation model of attachment. Pers Relat 19(3):445-464

48. Ştefan CA, Avram J (2017) Investigating direct and indirect effects of attachment on internalizing and externalizing problems through emotion regulation in a cross-sectional study. J Child Fam Stud 26(8):2311-2323

49. Slade A, Grienenberger J, Bernbach E, Levy D, Locker A (2005) Maternal reflective functioning, attachment, and the transmission gap: a preliminary study. Attach Hum Dev 7(3):283-298

50. Meins E, Fernyhough C, Fradley E, Tuckey M (2001) Rethinking maternal sensitivity: mothers' comments on infants' mental processes predict security of attachment at 12 months. J Child Psychol Psychiatry 42:637-648

51. Grienenberger JF, Kelly K, Slade A (2005) Maternal reflective functioning, mother infant affective communication, and infant attachment: exploring the link between mental states and observed caregiving behavior in the intergenerational transmission of attachment. Attach Hum Dev 7:299-311

52. Madigan S, Atkinson L, Laurin K, Benoit D (2013) Attachment and internalizing behavior in early childhood: a meta-analysis. Dev Psychol 49(4):672

53. McCartney K, Owen MT, Booth CL, Clarke-Stewart A, Vandell DL (2004) Testing a maternal attachment model of behavior problems in early childhood. J Child Psychol Psychiatry 45(4):765-778

54. Fearon RMP, Belsky J (2011) Infant-mother attachment and the growth of externalizing problems across the primary-school years. J Child Psychol Psychiatry 52(7):782-791

55. Groh AM, Roisman GI, van IJzendoorn MH, Bakermans-Kranenburg MJ, Fearon RP (2012) The significance of insecure and disorganized attachment for children's internalizing symptoms: a meta-analytic study. Child Dev 83(2):591-610

56. Chavira DA, Stein MB (2005) Childhood social anxiety disorder: from understanding to treatment. Child Adolescent Psychiatr Clin 14(4):797-818

57. Wood JJ (2006) Parental intrusiveness and children's separation anxiety in a clinical sample. Child Psychiatry Hum Dev 37(1):73-87

58. Hudson JL, Comer JS, Kendall PC (2008) Parental responses to positive and negative emotions in anxious and nonanxious children. J Clin Child Adolesc Psychol 37(2):303-313

59. Krink S, Muehlhan C, Luyten P, Romer G, Ramsauer B (2018) Parental reflective functioning affects sensitivity to distress in mothers with postpartum depression. J Child Fam Stud 27(5):1671-1681

60. Luyten P, Blatt SJ (2012) Psychodynamic treatment of depression. Psychiatr Clinics 35(1):111-129

61. Lam RW, Kennedy SH, McIntyre RS, Khullar A (2014) Cognitive dysfunction in major depressive disorder: effects on psychosocial functioning and implications for treatment. Can J Psychiatr 59(12):649-654

62. Israel BA, Schulz AJ, Parker EA, Becker AB (1998) Review of community-based research: assessing partnership approaches to improve public health. Annu Rev Public Health 19(1):173-202

63. Taubner S, Kessler H, Buchheim A, Kächele H, Staun L (2011) The role of mentalization in the psychoanalytic treatment of chronic depression. Psychiatr Interpersonal Biol Process 74(1):49-57

64. Pazzagli C, Delvecchio E, Raspa V, Mazzeschi C, Luyten P (2018) The parental reflective functioning questionnaire in mothers and fathers of school-aged children. J Child Fam Stud 27(1):80-90

65. Borelli JL, St John HK, Cho E, Suchman NE (2016) Reflective functioning in parents of school-aged children. Am J Orthopsychiatry 86(1):24

66. Fonagy P, Luyten P, Allison E (2015) Epistemic petrification and the restoration of epistemic trust: a new conceptualization of borderline personality disorder and its psychosocial treatment. J Pers Disord 29(5):575-609

67. Naghash F (2020) The moderator effect of The ADHD symptoms on the relationship between attachment dimensions and parental reflective functioning (Unpublished master's thesis). Shahid Beheshti University, Tehran, Iran

Publisher's Note Springer Nature remains neutral with regard to jurisdictional claims in published maps and institutional affiliations. 